\title{
Zur Kenntnis der komplexen anorganischen Säuren.
}

\author{
Von \\ F. Kehrmann. \\ IX. Abhandlung.
}

Über die Kieselwolframsäure, ihre Zusammensetzung nnd Analyse.

Nach gemeinsam mit Herrn B. FuÜrscheIM ${ }^{1}$ ausgeführten Versuchen.

Am Schlufse der 8. Mitteilung über komplexe anorganische Säuren ${ }^{2}$ habe ich der Vermutung Raum gegeben, dals die beiden von Marignac ${ }^{3}$ entdeckten und untersuchten Salzreihen der Silicoduodeciwolframsäure (acide silicotungstique) vielleicht nicht in der vom Entdecker angenommenen einfachen Beziehung neutraler zu sauren Salzen derselben Säure ständen, sondern dafs die sogenannten neutralen aus den sauren infolge einer Spaltung hervorgingen, analog denjenigen Spaltungen, welche die verschiedenen komplexen Phosphor- und Arsenwolframiate durch Säuren und Alkalien erleiden.

Gemeinsam mit Herm B. FuÜrscheim habe ich eine Versuchsreihe durchgeführt, um diese Vermutung mit Hilfe neuer analytischer Methoden zu prüfen. Das Ergebnis war eine Bestätigung unserer Erwartung. Es zeigte sich, dals das von Marignac für ein neutrales Salz der Kieselwolframsäure gehaltene Kaliumsalz, für welches sein Entdecker die Formel $4 \mathrm{H}_{2} \mathrm{O} . \mathrm{SiO}_{2} \cdot 12 \mathrm{WO}_{3}+$ aq. aufgestellt hat, tatsächlich einer anderen Reihe angehört und die Zusammensetzung

1 Berrahard Flürscheim, Inaug.-Dissertation, Heidelberg 1901, Druckerei J. HörNivg.

${ }^{2}$ Z. anorg. Chem. 22 (1899), 285.

3 Ann. Chim. Phys. [4] 3, 8. 
$7 \mathrm{~K}_{2} \mathrm{O} .2 \mathrm{SiO}_{2}, 20 \mathrm{WO}_{3}$ besitzt. Seine Bildung aus dem sogenannten sauren kieselwolframsauren Kalium $2 \mathrm{~K}_{2} \mathrm{O} . \mathrm{SiO}_{2} .12 \mathrm{WO}_{3}+$ aq. durch Einwirkung von Kaliumkarbonat ist die Folge eines Spaltungsvorganges und entspricht der folgenden Gleichung:

$$
\begin{gathered}
2\left(2 \mathrm{~K}_{2} \mathrm{O} . \mathrm{SiO}_{2} .12 \mathrm{WO}_{3}\right)+7 \mathrm{~K}_{2} \mathrm{CO}_{3}=7 \mathrm{~K}_{2} \mathrm{O} .2 \mathrm{SiO}_{2} .20 \mathrm{WO}_{3}+ \\
4 \mathrm{~K}_{2} \mathrm{WO}_{4}+7 \mathrm{CO}_{2} .
\end{gathered}
$$

Zur Trennung und Bestimunung von Wolframsäure und Kieselsäure erwies sich nach einer Anzahl vergeblicher Versuche dieses auf andere Weise zu bewirken, ${ }^{1}$ zuletzt der Weg als recht gut gangbar, welchen auch Marigarac einzuschlagen versucht, indessen als nicht zum Ziel führend verlassen hat, nämlich sehr oft wiederboltes Abrauchen der gemischten Oxyde mit wässeriger Flufssäure. Die Trennung der komplexen Säuren von den Alkalien gelang sehr befriedigend durch Fällen mit Chinolinchlorhydrat. ${ }^{2}$ Durch Glühen des Chinolinsalzes erhielt man die Summe von $\mathrm{WO}_{3}+\mathrm{SiO}_{2}$, in welcher durch Abrauchen mit Fluorwasserstoffsäure bis zu konstantem Gewicht die Wolframsäure direkt und die Kieselsäure aus dem Gewichtsverlust bestimmt wurde.

Mit Hilfe dieser Methoden gelang es uns, die von Marignac für die Kieselwolframsäure und ihr sogenanntes saures Kaliumsalz aufgestellten Formeln zu bestätigen, während, wie bereits erwähnt, für das sogenannte neutrale Salz, für welches sein Entdecker die Formel $4 \mathrm{~K}_{2} \mathrm{O} . \mathrm{SiO}_{2} .12 \mathrm{WO}_{3}$ annahm, die oben angeführte abweichende Zusammensetzung ermittelt wurde. Ferner gelang es, die beiden Spaltungsvorgänge, welche bei der Einwirkung von Alkalien auf die Salze $2 \mathrm{R}_{2} \mathrm{O} . \mathrm{SiO}_{2} .12 \mathrm{WO}_{3}$, und von Säuren auf die Salze $7 \mathrm{R}_{2} \mathrm{O} .2 \mathrm{SiO}_{2} \cdot 20 \mathrm{WO}_{3}$ stattfinden, durch qualitative, und wo ausführbar, auch quantitative Ermittelung der Spaltungsprodukte experimentell nachzuweisen.

\section{Experimenteller Teil.}

Es soll hier nicht auf die Einzelheiten der Ausarbeitung und Prüfung der benutzten analytischen Methoden eingegangen werden. Dieselben finden sich in der Dissertation von Herrn FuÜRscherm, ${ }^{3}$ auf welche hier verwiesen werden kann.

${ }^{1}$ Näheres über diese Versuche findet sich in der Dissertation von FLẗRSCHEM 1. c. S. $11-20$.

2 Z. anorg. Chem. 4 (1893), 142-143.

3 1. c. 


\section{I. Über die Kieselwolframsäure (acide silicotungstique) oder Kiesel- duodeci-wolframsäure $2 \mathrm{H}_{2} \mathrm{O} \cdot \mathrm{SiO}_{2} \cdot 12 \mathrm{WO}_{3}+$ aq.}

\section{A. Die freie Säure.}

Zur Analyse kam ein vom Eintdecker herrührendes prächtig kristallisiertes Präparat aus der Sammlung des Universitätslaboratoriums in Genf, welches nochmals aus wenig heilsem Wasser umkristallisiert und durch Erhitzen aut gelinde Rotglut bis zu konstantem Gewicht entwässert worden war. Um die Kieselsäure aus dem so erhaltenen Gemisch durch Abrauchen mit rückstandsfreier Flufssäure vollkommen zu entfernen, erwies es sich notwendig, nach je drei bis viermaligen Abdampfen, den etwas gesinterten Tiegelinhalt zu zerreiben und dann dessen Gewicht neu zu bestimmen, um den dadurch entstandenen allerdings geringen Gewichtsverlust in Abzug bringen zu können. War das zu analysierende Gemisch der Oxyde durch Glühen des Chinolinsalzes erhalten worden, so zeigte sich, dals weit weniger oft wiederholtes Abrauchen zur Erreichung der Gewichtskonstanz nötig war. Das Oxydgemisch aus dem weiter unten erwähnten, an $\mathrm{SiO}_{2}$ reichern Chinolinsalz, verhielt sich dagegen umgekehrt, so dafs hier sehr oft wiederholtes Abrauchen (30 bis $40 \mathrm{mal}$ ) und Zerkleinern erforderlich war.

I. $4.6452 \mathrm{~g}$ geglühte Säure verloren nach 11 maligem $\mathrm{Ab}$ rauchen und 6 maligem Pulvern $0.0971 \mathrm{~g}$ an Gewicht $=2.09 \%$.

II. $3.3198 \mathrm{~g}$ geglühtes Chinolinsalz verloren nach 5 maligem Abrauchen und 2 maligem Pulvern $0.0709 \mathrm{~g}=2.136 \%$.

III. $2.4359 \mathrm{~g}$ geglühtes Chinolinsalz verlor nach 11 maligem Abrauchen und 1 maligem Pulvern $0.0511 \mathrm{~g}=2.10 \%$.

Berechnet für $\mathrm{SiO}_{2}+12 \mathrm{WO}_{3}$ :

Gefunden:

$$
\begin{array}{rrrc}
\mathrm{WO}_{8}=97.88 & 97.91 & 97.864 & 97.90 \% \\
\mathrm{SiO}_{2}=2.12 & 2.09 & 2.136 & 2.10
\end{array}
$$

Das analysierte Chinolinsalz war durch Fällen der wässerigen Lösung der reinen Säure mit überschüssigem Chinolinchlorbydrat, Auswaschen mit Wasser, Trocknen und Glühen erhalten.

Hiermit erscheint die schon von Marignac gefundene Zusammensetzung der freien Säure bestätigt.

B. Das sogenannte saure kieselwolframsaure Kalium.

Nach Marignac $2 \mathrm{~K}_{2} \mathrm{O} .2 \mathrm{H}_{2} \mathrm{O} . \mathrm{SiO}_{2} \cdot 12 \mathrm{WO}_{3}+$ aq.

Zur Darstellung eines analysenreinen Salzes wurden $20 \mathrm{~g}$ des von MARIGNac herrührenden Präparates der freien Säure in wenig 
heifsem Wasser gelöst und die Lösung mit $5 \mathrm{~g}$ Chlorkalium, ebenfalls gelöst in heifsem Wasser, versetzt. Nach dem Abkühlen wurde das in kaltem Wasser schwerlösliche Salz abgesaugt und mit destilliertem eiskalten Wasser bis zum Verschwinden der Chlorreaktion gewaschen. Das Salz wurde dann einmal aus siedendem Wasser umkristallisiert und über Schwefelsäure getrocknet.

I. $2.0570 \mathrm{~g}$ verloren bei Rotglut $0.1935 \mathrm{~g}$ Wasser $=9.407 \%$.

II. $2.3615 \mathrm{~g}$ wasserhaltiges Salz wurde gleichzeitig mit dem zur Ermittelung des Wassergehaltes bestimmten Anteil abgewogen, in Wasser gelöst und mit Chinolinchlorhydrat gefällt. Dann wurde filtriert und der Niederschlag mit chinolinsalzbaltigem Wasser gut ausgewaschen, mit dem Filter verascht und unter Zusatz von etwas reiner rauchender Salpetersäure bis zu konstantem Gewicht geglüht. Filtrat und Waschwasser wurden vereinigt, in einer Platinschale eingedampft und schwach geglüht; dann nochmals in Wasser gelöst, zur Entfernung von etwas Kohle filtriert, nochmals in der Platinschale eingedampft und schliefslich bei kaum sichtbarer Rotglut konstant geglüht.

$2.3615 \mathrm{~g}$ ergaben $2.0100 \mathrm{SiO}_{2}+\mathrm{WO}_{3}$ und $0.2132 \mathrm{KCl}$.

Auf entwässertes Salz berechnet ergibt dieses:

$\begin{array}{lr}\text { Ber. für } 2 \mathrm{~K}_{2} \mathrm{O} . \mathrm{SiO}_{2} .12 \mathrm{WO}_{3}: & \text { Gefunden: } \\ \mathrm{K}_{2} \mathrm{O} \quad=6.22 & 6.29 \% \\ \mathrm{SiO}_{2}+\mathrm{WO}_{3}=93.78 & 93.95\end{array}$

Auch hier bestätigte sich die von MarJaNaC für sein saures K-Salz gefundene Zusammensetzung. Wie sich aus dem nachfolgenden ergibt, ist dasselbe jedoch kein saures Salz, da es nicht gelingt, ein an Basis reicheres Salz ohne Spaltung des Moleküls der Säure daraus darzustellen.

C. Das sogenannte normale kieselwolframsaure Kalium. Nach MarignaC $4 \mathrm{~K}_{2} 0 . \mathrm{SiO}_{2} \cdot 12 \mathrm{WO}_{3}+\mathrm{aq}$.

Von diesem Salz lag ebenfalls ein sehr reines von MarrganaC herrührendes Präparat vor, von dem ein Teil gepulvert und zu den nachstehenden Analysen verwendet wurde.

I. $2.2348 \mathrm{~g}$ verloren durch Glühen $0.1646 \mathrm{~g} \mathrm{H}_{2} \mathrm{O}=7.36 \%$. $5.4484 \mathrm{~g}$ wasserhaltiges $\mathrm{Salz}$ gaben $4.4344 \mathrm{~g}\left(\mathrm{SiO}_{2}+\mathrm{WO}_{3}\right)$ und $0.9900 \mathrm{~g} \mathrm{KCl}=0.6257 \mathrm{~g} \mathrm{~K}_{2} \mathrm{O}$. 
II. $1.9992 \mathrm{~g}$ verloren durch Glühen $0.1465 \mathrm{~g} \mathrm{H}_{2} \mathrm{O}=7.33 \%$, $5.6736 \mathrm{~g}$ wasserhaltiges $\mathrm{Salz}$ gaben $4.6158 \mathrm{~g}\left(\mathrm{SiO}_{2}+\mathrm{WO}_{3}\right)$ und $1.0282 \mathrm{~g} \mathrm{KCl}=0.6498 \mathrm{~g} \mathrm{~K}_{2} \mathrm{O}$.

Berechnet nach

Marianacs Formel:
I II

Gefunden:

$$
\begin{aligned}
& \begin{array}{llll}
\mathrm{K}_{2} \mathrm{O}=11.71 \quad \mathrm{~K}_{2} \mathrm{O} & =12.39 \quad 12.35 \%
\end{array} \\
& \left(\mathrm{SiO}_{2}+\mathrm{WO}_{3}\right)=88.3 \quad\left(\mathrm{SiO}_{2}+\mathrm{WO}_{3}\right)=87.85 \quad 87.79
\end{aligned}
$$

Berechnet für $7 \mathrm{~K}_{2} 0.2 \mathrm{SiO}_{2} .20 \mathrm{WO}_{3}$ :

$$
\begin{array}{ll}
\mathrm{K}_{2} \mathrm{O} & =12.18 \% \\
\left(\mathrm{SiO}_{2}+\mathrm{WO}_{3}\right) & =87.82
\end{array}
$$

Die erhaltenen Zahlen stimmen, wie man sieht, nicht gut mit der von MARIGNAC aufgestellten Formel, dagegen sehr befriedigend auf die Formel $7 \mathrm{~K}_{2} \mathrm{O} .2 \mathrm{SiO}_{2} .20 \mathrm{WO}_{3}$, wonach das Verbältnis der beiden Oxyde in dem sogenannten normalen Salze gegenüber dem sogenannten sauern zugunsten der Kieselsäure verschoben erscheint.

Die Entscheidung mufste daher durch eine möglichst genaue Bestimmung dieses Verbältnisses zu erbringen sein. Dazu diente der Glührückstand des Chinolinsalzes, welches zur Trennung der komplexen Säure vom Kalium in MarIGNaCs normalem Salz gedient hatte. Wie bereits erwähnt, wurde hier Gewichtskonstanz nur nach sehr oft wiederholtem Abrauchen mit Flufssäure erreicht.

I. $2.9867 \mathrm{~g}$ verloren nach 25 maligem Abrauchen und 3 maligem Pulvern $0.0750 \mathrm{~g} \mathrm{SiO}_{2}$.

II. $3.5339 \mathrm{~g}$ verloren nach 34 maligem Abrauchen und wiederholtem Pulvern $0.0888 \mathrm{~g} \mathrm{SiO}_{2}$.

Ber, f. d. Formel $4 \mathrm{~K}_{2} \mathrm{O} \cdot \mathrm{SiO}_{2} \cdot 12 \mathrm{WO}_{3}$ bezogen auf $\mathrm{SiO}_{2}+\mathrm{WO}_{3}$ :

Gefunden :

$$
\mathrm{SiO}_{2}=2.32
$$

Ber. f. d. Formel $7 \mathrm{~K}_{2} \mathrm{O} .2 \mathrm{SiO}_{2} \cdot 20 \mathrm{WO}_{3}$ bezogen auf $\mathrm{SiO}_{2}+\mathrm{WO}_{3}$ :

$$
\mathrm{SiO}_{2}=2.537 \quad \mathrm{SiO}_{2}=2.513
$$$$
\mathrm{SiO}_{2}=2.511 \%
$$

Gefunden:

II

Die neue Formel ersebeint also bestätigt. Da Marignacs vermeintliches normales Salz durch Einwirkung von Kaliumkarbonat auf eine Lösung von $2 \mathrm{~K}_{2} \mathrm{O} \cdot \mathrm{SiO}_{2} .12 \mathrm{WO}_{3}+$ aq. erhalten wird, so muls man demnach hier nicht einfach Neutralisation, sondern Spaltung annehmen und zwar Spaltung entsprechend der Gleichung:

$$
\begin{gathered}
2\left(2 \mathrm{~K}_{2} \mathrm{O}_{2} \mathrm{SiO}_{2} .12 \mathrm{WO}_{3}\right)+7 \mathrm{~K}_{2} \mathrm{CO}_{3}=7 \mathrm{~K}_{2} \mathrm{O} .2 \mathrm{SiO}_{2} .20 \mathrm{WO}_{3}+ \\
4 \mathrm{~K}_{2} \mathrm{WO}_{3}+7 \mathrm{CO}_{2} .
\end{gathered}
$$


Hiernach mufs in der Mutterlauge, aus welcher das Salz $7 \mathrm{~K}_{3} 0.2 \mathrm{SiO}_{2} .20 \mathrm{WO}_{3}$ auskristallisiert, Kaliumwolframiat nachweisbar sein, was, wie wir weiter unten sehen werden, in der Tat der Fall ist.

II. Marignacs wolframkieselsaures Kalium (acide tungstosilicique).

Der Entdecker betrachtet dieses Salz als isomer mit dem Kaliumsalz der Kieselwolframsäure (acide silicotungstique). Die nachstehend mitgeteilte Analyse bestätigt diese Auffassung.

Zur Untersuchung gelangte ein in grofsen hellgelben, vierseitigen Tafeln kristallisiertes Präparat aus der Sammlung des Genfer Universitäts-Laboratoriums.

$1.1086 \mathrm{~g}$ gaben $0.0572 \mathrm{~g}$ Wasser $a b=5.16 \%$.

$3.5844 \mathrm{~g}$ ergaben $0.3339 \mathrm{~g} \mathrm{KCl}=0.2110 \mathrm{~g} \mathrm{~K}_{2} \mathrm{O}$ und $3.2026 \mathrm{~g}$ $\left(\mathrm{SiO}_{2}+\mathrm{WO}_{3}\right)$.

$2.7032 \mathrm{~g}$ geglühtes Chinolinsalz verloren durch 18 maliges $\mathrm{Ab}$ rauchen und wiederholtes Pulvern $0.0587 \mathrm{~g}=2.17 \%$.

\begin{tabular}{llll}
\multicolumn{2}{r}{ Berechnet für $2 \mathrm{~K}_{2} \mathrm{O} . \mathrm{SiO}_{2} .12 \mathrm{WO}_{3}:$} & \multicolumn{2}{c}{ Gefunden: } \\
$\mathrm{K}_{2} \mathrm{O}$ & 6.22 & $\mathrm{~K}_{2} \mathrm{O}$ & $=6.21 \%$ \\
$\left(\mathrm{SiO}_{2}+\mathrm{WO}_{3}\right)=93.78$ & $\left(\mathrm{WO}_{3}+\mathrm{SiO}_{2}\right)=94.21$ \\
$\mathrm{SiO}_{2}=2.12$ & $\mathrm{SiO}_{2}$ & $=2.17$
\end{tabular}

III. Spaltungen der Kieselwolframiate durch Säuren und Alkalien.

A. Einwirkung von Kaliumkarbonat auf kieselduodeciwolframsaures Kalium.

$4.8315 \mathrm{~g}$ des Marignacschen Präparates ${ }^{1}$ verloren $0.4530 \mathrm{~g}$ Wasser $=9.37 \%$.

$29.9552 \mathrm{~g}$ wurden in einem kleinen Kolben in der eben hinreichenden Wassermenge gelöst. Nun wurde eine kalte Lösung von $10 \mathrm{~g}$ Kaliumkarbonat (von $10 \%$ Wassergehalt) in $55 \mathrm{ccm}$ Wasser in Portionen von je $5.5 \mathrm{ccm}$ hinzugefügt, nach jedem Zusatz umgeschüttelt und gewartet, bis die Ausscheidung glitzernder Kristallkrusten des vermeintlichen normalen Salzes nicht weiter zunahm, und die Lösung neutral auf Lackmus reagierte. Hierzu waren im Ganzen $4 \mathrm{mal} 5.5 \mathrm{ccm}$ und noch $1 \mathrm{ccm}$, zusammen $23 \mathrm{ccm}$ erforderlich.

1 Das sich in der Sammlung befindende Präparat war stark verwittert und wurde einmal aus Wasser umkristallisiert. 
Die ausgeschiedenen Kristalle wurden von der Mutterlauge getrennt, etwas mit kaltem Wasser abgewaschen, und in einer Platinschale bei $120^{\circ}$ getrocknet. Sie wogen $22.8597 \mathrm{~g}$ und enthielten noch $5.8 \%$ Wasser. In den Eigenschaften zeigte sich das Salz absolut identisch mit einem von Marignac stammenden Präparat, welches als $4 \mathrm{~K}_{2} \mathrm{O} \cdot \mathrm{SiO}_{2} \cdot 12 \mathrm{WO}_{3}+$ aq. bezeichnet war, und dessen Zusammensetzung von uns zu $7 \mathrm{~K}_{2} \mathrm{O} .2 \mathrm{SiO}_{2} .20 \mathrm{WO}_{3}+$ aq. ermittelt worden war.

Die von den Kristallen getrennte Mutterlauge wurde auf $200 \mathrm{ccm}$ mit Wasser verdünnt und in zwei Hälften geteilt. Die eine diente zum Nachweise von Kaliumwolframiat, die andere zur Bestimmung der noch in Lösung befindlichen $\left(\mathrm{SiO}_{2}+\mathrm{WO}_{3}\right)$.

$100 \mathrm{ccm}$ wurden in einer Porzellanschale mit Salzsäure eingedampft; hierbei schied sich gelbe Wolframsäure in reichlicher Menge aus. Dieselbe wurde abfiltriert, mit heifser verdünnter Salzsäure und Wasser ausgewaschen, geglüht und gewogen. Der Rückstand wog 0.7772 g. Hiermit war qualitativ die Abspaltung von Wolframsäure beim "Neutralisieren" des "sauren" Duodecisalzes bewiesen. Das Filtrat von der Wolframsäure gab durch Fällen mit Chinolinchlorhydrat und Veraschen noch $2.4690 \mathrm{~g}\left(\mathrm{SiO}_{2}+\mathrm{WO}_{3}\right)$. Dieselben rührten von dem in Lösung gebliebenen Anteil des Salzes $7 \mathrm{~K}_{2} \mathrm{O}$. $2 \mathrm{SiO}_{2} .20 \mathrm{WO}_{3}$ her. Die Totalsumme der erhaltenen Oxyde betrug demnach $3.2462 \mathrm{~g}$.

$100 \mathrm{~cm}$ wurden nach dem Ansäuren mit Essigsäure und Erwärmung auf $60^{\circ}$ direkt mit Chinolinchlorhydrat gefällt. Der Niederschlag ist ein Gemisch von Chinolinwolframiat und Kieselwolframsaurem Chinolin. Nach dem Abfiltrieren, Auswaschen und Glühen wurden $3.3429 \mathrm{~g}$ Oxyde erhalten. Durch Abrauchen mit Flufssäure verloren diese $0.0465 \mathrm{~g}$ an Gewicht, entsprechend $1.42 \%$ Kieselsäure. Hiermit ist bewiesen, dafs neben dem durch die Spaltung bei der Neutralisation gebildeten Salz $7 \mathrm{~K}_{2} \mathrm{O} .2 \mathrm{SiO}_{2} .20 \mathrm{WO}_{3}$, welches $2.54 \%$ Kieselsäure verlangt, eine beträchtliche Menge Wolframsäure in Form von Wolframiat in Lösung sein mufste. Deren Gesamtmenge konnte in Versuch I durch Abrauchen mit Salzsäure deshalb nicht erhalten werden, weil sich das ebenfalls in der Lösung vorhandene $\mathrm{Salz} 7 \mathrm{~K}_{2} \mathrm{O} .2 \mathrm{SiO}_{2} .20 \mathrm{WO}_{3}$ hierbei unter Aufnahme von Wolframsäure in Duodeciwolframiat umwandelt, welches nur $2.12 \% \mathrm{SiO}_{2}$ verlangt. 
B. Einwirkung von Salzsäure auf das Salz.

$$
7 \mathrm{~K}_{2} 0.2 \mathrm{SiO}_{2} .20 \mathrm{WO}_{3}+\text { aq. }
$$

$20 \mathrm{~g}$ wurden fein gepulvert und in einer Platinschale tropfenweise unter Umrühren mit zweiprozentiger Salzsäure versetzt. Es trat bald schon in der Kälte völlig klare Auflösung ein. Eine Probe solcher Flüssigkeit gab auf Zusatz von festem Chlorammonium keinen Niederschlag, ein Beweis, dals dieselbe zunächst keine Duodecisäure enthält. Nach kurzem Aufkochen tritt Bildung der letzteren ein und das in Salmiaklösung sehr wenig lösliche Ammonsalz derselben scheidet sich grolsenteils schon in der Hitze aus. Nachdem die mit Salzsäure in der Kälte in Lösung gebrachten $20 \mathrm{~g}$ fünf Stunden gestanden hatten, war die Flüssigkeit zum steifen Kristallbrei erstarrt, welcher nach dem Zerreiben abgesaugt und mit wenig Eiswasser gewaschen wurde. Nach dem Lösen desselben in hoifsem Wasser blieb etwas $\mathrm{WO}_{3}$-haltige $\mathrm{SiO}_{2}$ zurück und zwar $0.0045 \mathrm{~g}$, welche aus $0.0034 \mathrm{~g} \mathrm{WO}_{3}$ und $0.0011 \mathrm{~g} \mathrm{SiO}_{2}$ bestand. Das Filtrat schied nach dem Erkalten ein Gemisch von Duodecisalz mit dem Salz $7 \mathrm{~K}_{2} \mathrm{O} .2 \mathrm{SiO}_{2} .20 \mathrm{WO}_{3}$ ab. Dasselbe wurde nun mit viel Salzsäure versetzt und zur Trockne verdampft. Nach dem Aufnehmen des Rückstandes in heifsem. Wasser blieben $0.0115 \mathrm{~g} \mathrm{SiO}_{2}$ und $0.0017 \mathrm{~g}$ $\mathrm{WO}_{3}$ zurück. Ferner lieferte das Filtrat von den oben erwähnten Kristallen bei gleicher Behandlung mit Salzsäure noch $0.0106 \mathrm{~g}$ $\mathrm{SiO}_{2}$ und $0.0036 \mathrm{~g} \mathrm{WO}$. Im Ganzen waren daher durch Bebandeln von $20 \mathrm{~g}$ kristallisiertem Salz $7 \mathrm{~K}_{2} \mathrm{O} .2 \mathrm{SiO}_{2} .20 \mathrm{WO}_{3}$ mit Salzsäure erhalten worden, $0.0232 \mathrm{~g} \mathrm{SiO}_{2}$ und $0.0087 \mathrm{~g} \mathrm{WO}_{3}{ }^{*}$. Aus den verschiedenen erhaltenen Laugen konnte durch fraktionierte Kristallisation eine bedeutende Menge Kaliumsalz der Kieselduodeciwolframsäure, ferner einige Kristalle des isomeren Salzes, in geringer Menge weifse nicht weiter untersuchte warzenförmige Kriställchen, und schlielslich Chlorkalium erhalten werden.

Nach vorstehendem ist sicher, dafs die Entstehung des Kaliumsalzes der Kieselduodeciwolframsäure durch Einwirkung von Säuren auf das $\mathrm{Salz} 7 \mathrm{~K}_{2} \mathrm{O} .2 \mathrm{SiO}_{2} .20 \mathrm{WO}_{3}$ von einer Abspaltung von $\mathrm{SiO}_{2}$ begleitet ist. Auffallend ist nur, dafs die so erhaltene $\mathrm{Menge}_{\mathrm{SiO}}$ weit hinter der theoretischen zurückbleibt.

\section{Ist Kieselsäure im Moment der Abscheidung aus ihren Salzen durch stärkere Säuren flüchtîg?}

Da eine bejahende Antwort auf diese Frage die Erklärung für das Manko an $\mathrm{SiO}_{2}$ in dem letztbeschriebenen Versuche liefern 
würde, hat Herr Fü̈rscherm die folgenden Versuche angestellt, deren Resultat sehr zugunsten der Flüchtigkeit sprechen.

I. $0.2579 \mathrm{~g}$ geglühte $\mathrm{SiO}_{2}$ wurde mit $1 \mathrm{~g} \mathrm{KNaCO}_{3}$ im Platintiegel geschmolzen, die Schmelze in heilsem Wasser gelöst und in einer Platinschale mit konzentriertem $\mathrm{HCl}$ zur Trockne verdampft. Dieses Eindampfen mit Salzsäure wurde $20 \mathrm{mal}$ wiederholt. Dann wurde mit heilsem Wasser aufgenommen, abfiltriert, gut ausgewaschen und geglüht. Erhalten $0.2538 \mathrm{~g} \mathrm{SiO}_{2}$, also weniger $0.0041 \mathrm{~g}$.

II. $0.2538 \mathrm{~g}$ wurden mit $1 / 2 \mathrm{~g} \mathrm{KNaCO}_{3}$ geschmolzen, zweimal mit Salzsäure abgeraucht und sonst wie in I. behandelt. Erhalten $0.2514 \mathrm{~g} \mathrm{SiO}_{2}$, also weniger $0.0024 \mathrm{~g}$.

III. $0.2497 \mathrm{~g}$ im Gebläse konstant geglühte $\mathrm{SiO}_{3}$ wurde wie unter II. behandelt. Erhalten $0.2470 \mathrm{~g}$, also weniger $0.0027 \mathrm{~g}$.

IV. $0.2470 \mathrm{~g}$ wie unter III behandelt, aber mit Salpetersäure abgeraucht. Erhalten $0.2457 \mathrm{~g}$, also weniger $0.0013 \mathrm{~g}$.

V. $0.2457 \mathrm{~g}$ wie unter IV. mit Salpetersäure behandelt. Erhalten $0.2432 \mathrm{~g}$, also weniger $0.0025 \mathrm{~g}$.

VI. $0.4894 \mathrm{~g}$ mit $1 \mathrm{~g} \mathrm{KNaCO}_{3}$ geschmolzen und fünf mal mit Salpetersäure abgeraucht. Erhalten $0.4839 \mathrm{~g}$, also weniger $0.0055 \mathrm{~g}$.

Im Filtrat und Waschwasser liefs sich bei keinem der Versuche nach Eindampfen und Aufnehmen mit wenig Wasser $\mathrm{SiO}_{2}$ in wägbarer Menge nachweisen.

Die Verluste konnten daher nicht wohl durch Lösen in Wasser bedingt sein.

Nach vorstehendem Resultate zu urteilen, scheint man zu der Annahme berechtigt zu sein, dafs Kieselsäure im Moment der Abscheidung aus ihren Salzen durch starke Mineralsäuren und Eindampfen sich in geringen, aber doch wägbaren Mengen verflüchtigt. Man wird, bei Bestätigung dieser Beobachtungen durch auf andere Weise durchzuführende Kontrollversuche, diese Flüchtigkeit wohl der bis heute hypothetischen monomolekularen $\mathrm{SiO}_{2}$ oder einem Hydrat derselben zuschreiben dürfen, welche dann sehr schnell durch Polymerisation, bezw. durch Bildung von Polykieselsäure verloren geht. Die Beziehungen der Kieselsäure zur Kohlensäure und Borsäure würden sich dann von einer neuen Seite zeigen. 
Da weiter vorn gezeigt ist, dafs bei wiederholtem Abdampfen des Salzes $7 \mathrm{~K}_{2} 0.2 \mathrm{SiO}_{2} .20 \mathrm{WO}_{3}$ mit überschüssiger Salzsäure die Kieselsäure sich sehr allmählich abscheidet, also als andauernd im Entstehungszustande befindlich angesehen werden darf, so ist leicht einzusehen, dafs, im Falle der Flüchtigkeit dieser Säure, während dieses Zersetzungsvorganges die Bedingungen für deren Verflüchtigung besonders günstig liegen. Damit fände der verhältnismälsig grofse Verlust an $\mathrm{SiO}_{2}$ seine Erklärung.

Die Untersuchung der Silicowolframsäuren wird fortgesetzt werden.

Genf, Universitätslaboratorium, April 1902.

Bei der Redaktion eingegangen am 14. Januar 1904. 